\title{
Subscapular and Pectoralis Major Sparing Deltopectoral Approach for Reverse Total Shoulder Arthroplasty
}

\author{
Young-Woo Chung, Jae-Woong Seo, Ki-Yong $A n^{\varpi}$ \\ Department of Orthopedic Surgery, Gwangju Veterans Hospital, Gwangju, Korea
}

In reverse ball shoulder replacement, surgery is usually performed using a deltopectoral approach or an anterosuperior transdeltoid approach. The deltopectoral approach is to incise the pectoralis major to upper $1 / 3$ to $1 / 2$, and subscapularis tendon should be removed at the lesser tuberosity of the humerus. This approach has the problem of breaking the shoulder deltoid instead of incising the rotator cuff. Therefore, we report a detailed procedure of reverse ball shoulder replacement using approach without incision of the pectoralis major muscle and subscapularis muscle.

(Clin Shoulder Elbow 2019;22(2):110-112)

Key Words: Reverse ball shoulder replacement; Deltopectoral approach; Anterosuperior transdeltoid approach

\section{Introduction}

Reverse ball shoulder replacement is usually performed using the deltopectoral approach or anterosuperior transdeltoid approach. The deltopectoral approach requires the incision of the insertion of the pectoralis major to the upper $1 / 3$ to $1 / 2$, and the detachment of the subscapularis from the lesser tuberosity of the humerus. Although the anterosuperior transdeltoid approach does not involve the incision of the rotator cuff in the anterior portion of the shoulder joint, it requires the incision of the deltoid muscle. Therefore, we performed reverse total shoulder arthroplasty by taking the deltopectoral approach without the incision of the pectoralis major and subscapularis muscles, and report the detailed procedures of this surgical method.

\section{Technique}

After the patient is placed in a beach-chair position, the coracoid process and cephalic vein of the patient are palpated and marked as in the deltopectoral approach, a skin incision is made more distally by about $5 \mathrm{~mm}$ than in the deltopectoral approach.
After a deep incision is made by pulling the cephalic vein inward, the proximal part of the subscapularis is examined and if there is any remaining biceps tendon long head, it is examined to identify the rotator interval. If there is any remaining biceps tendon long head, it is incised and the remaining synovium and joint membrane of the humeral head are removed to expose the humeral head upward (Fig. 1A). When the arm is extended, the humeral head is more exposed at the rotator interval, and if it is sufficiently seen, the elbow is pushed upward and the humeral head is exposed by pushing it up over the rotator interval (Fig. 1B, C). After the end of the humeral head and the area between the lesser tuberosity and the greater tuberosity are identified and marked, an intramedullary guide is placed along the humeral shaft with an owl. At this time, although the humerus shaft is not exposed, the osteotomy site is sufficiently exposed and when a rod is inserted, the inclination of the site can be checked. Osteotomy is performed using an oscillating saw, taking care not to cause damage to the anterior portion of the subscapular muscle and the remaining posterior portion of the rotator cuff. When osteotomy is performed appropriately, the tip of the Hohmann retractor is placed on the inferior pole of the glenoid cavity, and

Received September 9, 2018. Revised January 14, 2019. Accepted January 21, 2019.

Correspondence to: Ki-Yong An

Department of Orthopedic Surgery, Gwangju Veterans Hospital, 99 Cheomdanwolbong-ro, Gwansan-gu, Gwangju 62284, Korea

Tel: +82-62-602-6162, Fax: +82-62-602-6164, E-mail: mdaky@hanmail.net, ORCID: https://orcid.org/0000-0003-3583-6425

IRB approval (exemption granted): Gwangju Veterans Hospital (No. 2019-11-1).

Financial support: None. Conflict of interests: None. 

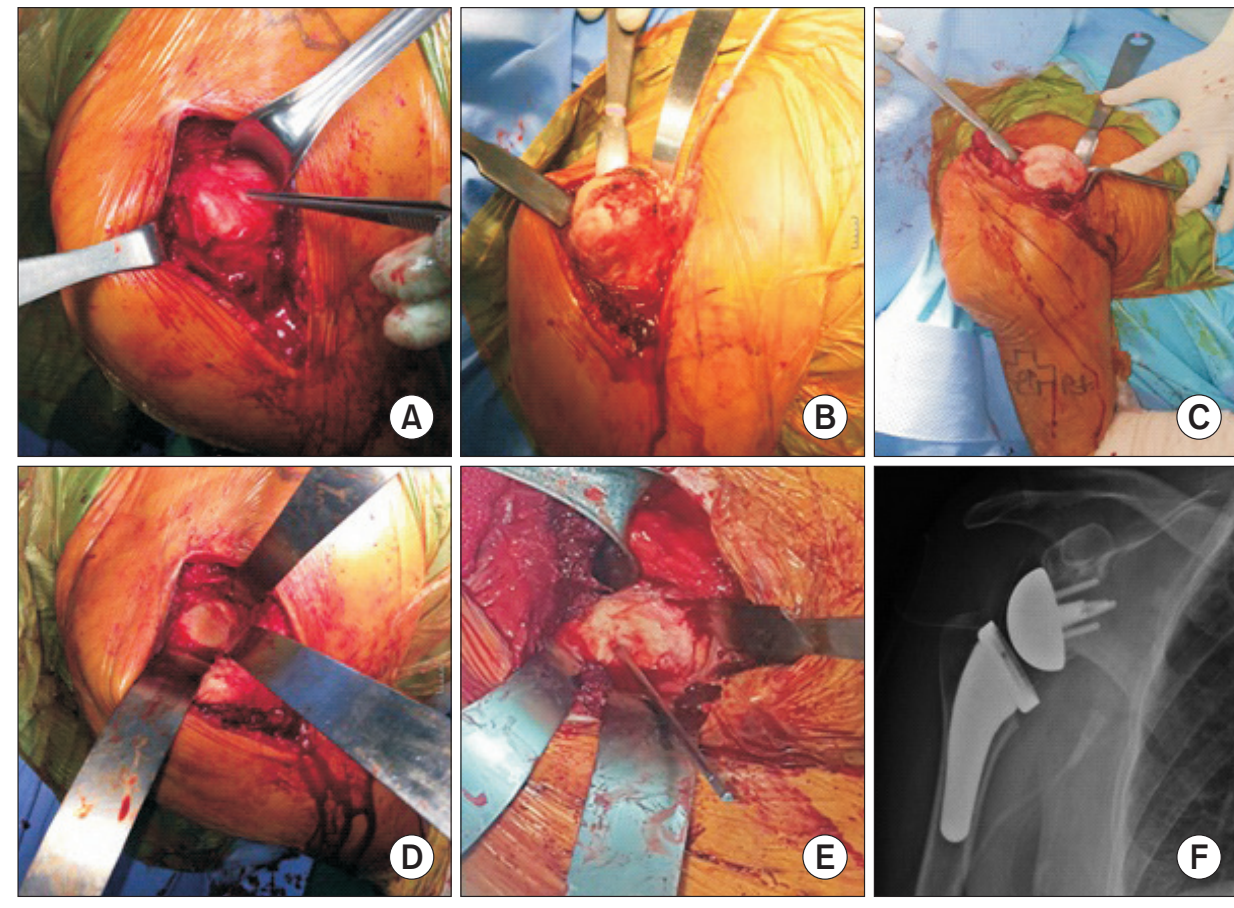

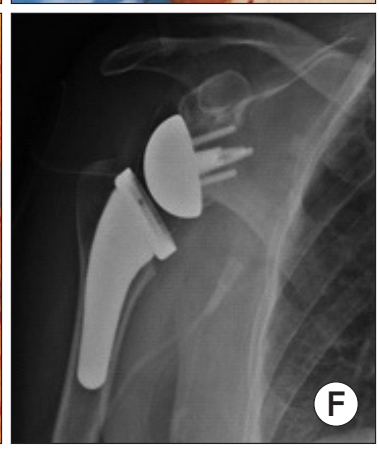

Fig. 1. Representative perioperative images of the deltopectoral approach to reverse total shoulder arthroplasty. (A) After confirmation of the rotator interval and resection of the upper arm, during which the biceps tendon long head remains intact, the remaining synovium and joints of the humeral head are removed, and the humeral head is exposed upward. (B, C) When the arm is extended, the humerus head is exposed at the rotator interval, and, if space is sufficient, the elbow is pushed upward and the humerus head is pushed up through the space within the rotator interval. (D, E) When and appropriate level of osteotomy has been performed, the tip of a Hohmann retractor is placed on the inferior pole of the joint and the osteotomized humerus is pushed downward along the humerus shaft, while the Hohmann retractor is moved forward and backward to expose the glenoid fossa. (F) Postoperative $\mathrm{X}$-ray of completed shoulder arthroplasty. the osteotomized humeral head is pushed downward toward the humerus shaft, and retractors are placed anteriorly and posteriorly to expose the glenoid cavity (Fig. 1D, E). Then, after incising the glenoid labrum and removing the glenoid cartilage, a glenoid implant is positioned obliquely slightly below the center of the joint. After the glenoid sphere is inserted, the humeral head is pushed upward again and the humeral implant is inserted. Then, after the reduction of the shoulder joint is performed, the tension of the conjoint tendon and that of the deltoid are checked and suture is performed.

\section{Discussion}

Reverse total shoulder arthroplasty is a treatment method that can be used to relieve shoulder pain and restore the range of motion in patients with irreparable rotator cuff tears or rotator cuff tear arthropathy. ${ }^{1)}$ If the anterosuperior transdeltoid approach is used, the anterior deltoid needs to be detached from the acromion, so the anterosuperior transdeltoid approach has the disadvantage of affecting the active flexion of the shoulder joint due to the weakening of muscular strength of the anterior deltoid. In addition, it may cause axillary nerve injury if the deltoid muscle is excessively pulled distally in order to obtain the view of the inferior portion of the glenoid cavity. If the deltopectoral approach is used, about the half of the pectoralis major is incised and when the humerus is rotated externally, the subscapularis tendon is completely detached from the lesser tuberosity of the humeral head to expose the humeral head, and artificial joint is inserted. Then, the incised subscapular muscles and pectoralis major are restored as much as possible, and surgery is completed. In the deltopectoral approach, a long skin incision should be made to the insertion of the pectoralis major on the humerus, and since the pectoralis major and the scapula are incised, they need to be restored after surgery. In addition to these drawbacks, there is a need for the time to keep the shoulder joint fixed with devices such as an abduction brace to prevent a retear. Moreover, since the whole humerus is exposed by rotating the arm externally, careful attention should be given to the axillary nerve, and tri-sister vessel coagulation of the anterior humeral circumflex artery may be required. In the case of irreparable rotator cuff tears, it is uncommon for the subscapularis to remain intact. Even in such cases, if the deltopectoral approach is used, it is necessary to completely detach the incomplete subscapularis from the insertion site on the humerus to gain access to the humeral head and glenoid cavity. If the subscapularis- and deltoid-sparing deltopectoral approach is used, operation time can be reduced because there is no need to restore them because total shoulder arthroplasty is performed by pushing up the humeral head of the rotator cuff toward the rotator interval without incising the subscapularis and pectoralis muscle. It has been suggested that if total shoulder arthroplasty is performed, exercised should be performed as soon as possible to minimize joint stiffness, and some researchers have claimed that the shoulder joint should be fixed by using an abduction brace for 6 weeks $^{3,4}$ in order to reduce excessive tension applied to the restoration area of the subscapularis. In contrast, in the subscapularis-sparing approach used in this study, since the subscapularis is not incised, there is no need to consider the 
excessive tension of the subscapularis and only the arm sling is maintained. As a result, the exercises to restore the range of motion can be performed more quickly. In addition because the subscapularis and pectoral muscles are not incised and thus a skin incision is not made downward along the humerus, another advantage of this approach is small skin incisions. However, because incision sizes are small and the whole humeral head is not visible, it is difficult to identify directions, so it is difficult for a beginner to perform it easily. It has been reported that even when total shoulder arthroplasty was performed by a similar method, there were side effects, such as mismatch or malposition of the humeral head, and residual osteophytes in the humeral head. ${ }^{5)}$ In addition, this approach is difficult to use when total shoulder arthroplasty is performed in patients with four part fractures of the humerus. Because the axillary nerve is difficult to identify during surgery, caution is needed when performing osteotomy with an oscillating saw. In the case of the shoulder joint with a loss of active abduction function, reverse total shoulder arthroplasty and tendon transfer of the latissimus dorsi and teres major are necessary ${ }^{6)}$ but they are difficult to apply in this surgical approach.

In conclusion, the deltopectoral approach without the incision of the subscapularis and pectoralis major muscle used in reverse total shoulder arthroplasty can be performed without causing damage to the subscapularis and pectoralis major muscles, thus reducing the time required for restoration of the scapular and pectoral muscle, and it Is a useful surgical method that does not require fixation of the shoulder joint during rehabilitation.

\section{References}

1. Sirveaux F, Favard L, Oudet D, Huquet D, Walch G, Molé D. Grammont inverted total shoulder arthroplasty in the treatment of glenohumeral osteoarthritis with massive rupture of the cuff. Results of a multicentre study of 80 shoulders. J Bone Joint Surg Br. 2004;86(3):388-95.

2. Chung SW, Kim JY, Oh JH. Reverse total shoulder arthroplasty: techniques and pitfalls. Clin Should Elbow. 2011;14(1):12533. doi: 10.5397/CiSE.2011.14.1.125.

3. Brems JJ. Rehabilitation after total shoulder arthroplasty: current concepts. Semin Arthroplast. 2007;18(1):55-65. doi: 10.1053/j.sart.2006.11.001.

4. Oh JH, Song BW. The current state of total shoulder arthroplasty. Clin Should Elbow. 2011;14(2):253-61. doi: 10.5397/ CiSE.2011.14.2.253.

5. Lafosse L, Schnaser E, Haag M, Gobezie R. Primary total shoulder arthroplasty performed entirely thru the rotator interval: technique and minimum two-year outcomes. J Shoulder Elbow Surg. 2009;18(6):864-73. doi: 10.1016/j.jse. 2009.03.017.

6. Boileau P, Chuinard C, Roussanne Y, Bicknell RT, Rochet N, Trojani C. Reverse shoulder arthroplasty combined with a modified latissimus dorsi and teres major tendon transfer for shoulder pseudoparalysis associated with dropping arm. Clin Orthop Relat Res. 2008;466(3):584-93. doi: 10.1007/s11999008-0114-x. 